\title{
Rauchstopp nun fester Teil des DMP COPD
}

- Wie bereits bericht, hat der Gemeinsame Bundesausschuss (G-BA) die Anforderungen an das Disease-Management-Programm (DMP) zur Behandlung von Patienten mit chronisch obstruktiver Lungenerkrankung (COPD) aktualisiert (MMW 16/2016, S. 24). Die Änderungen wurden nun am 14. Oktober 2016 im Bundesanzeiger veröffentlicht und können wie geplant am 1. Januar 2017 in Kraft treten. Betroffen von der Neuerung sind in der DMP-Anforderungen-Richtlinie des G-BA die COPD-Anlagen 11 und 12 (Anforderungen und Dokumentation).

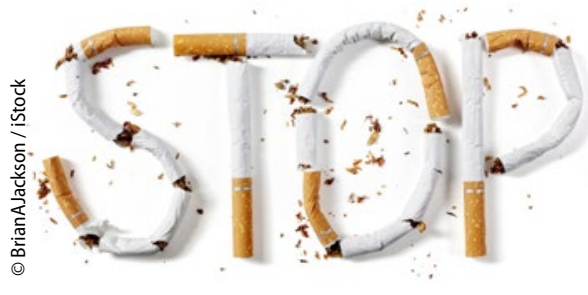

\section{MMW-KOMMENTAR}

Eine der wesentlichen Änderungen betrifft die stärkere Gewichtung der Tabakentwöhnung. Aktuell rauchende COPD-Patienten im DMP haben nun einen Anspruch auf ein strukturiertes, publiziertes Programm. Der G-BA hat die Vertragspartner beauftragt zu prüfen, ob die hierzulande verfügbaren Programme geeignet sind. Bisher genügen die folgenden Tabakentwöhnungsprogramme bereits diesen Anforderungen:

- Rauchfrei-Programm (IFT-Institut München, www.rauchfrei-programm.de),

- Nichtraucher in sechs Wochen (Universitätsklinik Tübingen, Prof. Batra),

- Mein Nichtraucherprogramm (Bundesverband der Pneumologen in Deutschland, www.pneumologenverband.de/37.html). Es können aber auch Programme vereinbart werden, die nicht bundesweit, sondern nur regional verfügbar sind - sofern sie validiert und publiziert sind.

\section{Hier steht eine Anzeige.}

